\title{
Computational design and prediction of solid-state properties of metal-organic frameworks
}

\author{
Mihails Arhangelskis ${ }^{1}$, Yizhi Xu${ }^{1}$, James P. Darby ${ }^{2}$, Novendra Novendra ${ }^{3}$, Joseph M. Marrett ${ }^{4}$, Athanassios D. \\ Katsenis ${ }^{4}$, Hatem M. Titi ${ }^{4}$, Andrew J. Morris ${ }^{5}$, Tomislav Friščić ${ }^{4}$, Alexandra Navrotsky, \\ ${ }^{1}$ University of Warsaw, Warsaw, Poland; ${ }^{2}$ Department of Engineering, University of Cambridge; ${ }^{3}$ Peter A. Rock Thermochemistry \\ Laboratory and NEAT ORU, University of California Davis; ${ }^{4}$ Department of chemistry, McGill University; ${ }^{5} 5$ School of Metallurgy \\ and Materials, University of Birmingham; ${ }^{6}$ School of Molecular Sciences and Center for Materials of the Universe, \\ Arizona State University; \\ m.arhangelskis@uw.edu.pl
}

Design of new types of metal-organic frameworks (MOFs), the microporous materials with a wide range of functional properties, is an active area of materials research. The vast variety of available linker and node combinations leads to an incredible variety of potential MOF structures, providing an opportunity for tailoring the functional properties and thermodynamic stability of the new materials. At the same time, navigating the vast structural space of putative MOFs is proving to be a challenge for experimental screening, that can benefit from the guidance provided by computational chemistry methods. In this presentation we will describe the application of periodic density-functional theory (DFT) calculations together with state-of-the-art ab initio crystal structure prediction (CSP) calculations in elucidating the structural aspects of MOF thermodynamic stability and performing computational property-driven design of new MOFs.

The presentation will commence with a theoretical study of the systematic effects of linker substituents on the thermodynamic stability of a series of isostructural zeolitic imidazolate frameworks (ZIFs) with sodalite topology.[1] We will show how periodic density functional theory (DFT) calculations offer highly accurate predictions for the thermodynamic stability of the ZIF structures as a function of linker substitution, also taking into account the effects of crystal packing of pure ligands. The accuracy of periodic DFT calculations will be backed up by the excellent correlation with the experimental solution calorimetry measurements. Moreover, it will be demonstrated how simple descriptors, such as Hammett $\sigma$-constants and electrostatic surface potentials (ESPs) offer convenient tools for rapid pre-screening of linker substituents, before performing a more in-depth computational analysis.

We will continue with a demonstration of our recently-developed method for ab initio CSP, which uses the Wyckoff Alignment of Molecules (WAM) procedure to predict the structures of new MOFs, with improved computational efficiency enabled through careful consideration of molecular and crystallographic symmetry.[2] We will demonstrate the use of our CSP approach for predicting polymorphism, thermodynamic stability, porosity and optical properties of new MOFs in a series of computational studies backed up by experiment. It will also be shown how MOF CSP can be used as a tool to elucidate the crystal structures of poorly-crystalline MOFs, which are difficult to determine with X-ray diffraction methods.

[1] Novendra, N., Marrett, J. M., Katsenis, A. D., Titi, H. M., Arhangelskis, M., Friščić, T. \& Navrotsky, A. J. (2020). J. Am. Chem. Soc. 142, 21720.

[2] Darby, J. P., Arhangelskis, M., Katsenis, A. D., Marrett, J. M., Friščić, T. \& Morris, A. J. (2020). Chem. Mater. $32,5835$.

Keywords: metal-organic frameworks; polymorph stability; crystal structure prediction; molecular descriptors 